\title{
SOME ASPECTS OF POSITRONIUM INTERACTION WITH ELEMENTARY FREE VOLUMES IN POLYMERS
}

\author{
V.P. Shantarovich \\ Semenov Institute of Chemical Physics, Russian Academy of Sciences \\ 4 Kosygin str., 117334, Moscow, Russia
}

\begin{abstract}
This paper contains a discussion of original and recently published results on positronium annihilation lifetime studies of elementary free volumes in the group of polymer glasses. The results are used for indication of non-homogeneity of these materials and give some basis for considering a connection of the long-lived component intensities $I_{3}\left(I_{4}\right)$ with concentrations $N_{3}\left(N_{4}\right)$ of elementary free volumes and, in this way, with fractional free volume.
\end{abstract}

PACS numbers: 78.70.Bj

\section{Introduction}

Today, positronium annihilation lifetime (PAL) spectroscopy can be considered as the most direct probe method to estimate free volumes in polymers. The longest lifetime component(s) $\tau_{3}\left(\tau_{4}\right)$, being characteristic of annihilation in the bound state $\mathrm{e}^{+} \mathrm{e}^{-}$(a hydrogen-like ortho-positronium atom o-Ps) is indicative of free volume in material [1-4]. Large number of papers are devoted to this problem. We are not going to discuss here the whole spectrum of the studies in this field and refer the interested reader to the book [5] and to some of the recent publications of more or less general character [6-8]. In the scope of the mechanism of non-instantaneous trapping of Ps by elementary free volumes (EFV) [6, 9], which seems to be supported now by the recent angular-momentum-correlation (AMOC) experiments [10], we shall demonstrate the approach to quantitative analysis of PAL experimental results for a large number of polymer glasses (Table 1, [11]) with different free volumes. Our results [11] on o-Ps quenching by $\mathrm{O}_{2}$ without inhibition of Ps formation in some polymer glasses give the first direct evidence of non-homogeneity of these materials. Recent discussion on this topic by Fujii and Rigby is mentioned in [11]. Indication on existence of complex size-distribution of free volume elements was obtained using both finite-term and continuous procedures of the data treatment. A smooth transition was observed from unimodal to 
obviously bimodal distributions in connection with permeability and selectivity of polymers for the light gases. Using these results we shall calculate here the densities of elementary free volumes $N_{3}$ and $N_{4}$ in the two approaches: homogeneous and heterogeneous distributions of the EFV in polymers. Here and later on, the EFV concentration $N_{i}$ is labeled with a subscript showing the number of the corresponding lifetime component. The results enable calculations of the specific free volumes $\left(V_{\mathrm{f} 3}=N_{3} v_{\mathrm{f} 3} ; V_{\mathrm{f} 4}=N_{4} v_{\mathrm{f} 4} ; V_{\mathrm{f}}=V_{\mathrm{f} 3}+V_{\mathrm{f} 4}\right)$ and fractional free volumes. They will correlate with permeation and selectivity of corresponding polymers and with calculations by the Bondi method. Our kinetic consideration of Ps trapping by EFV infers that the trapping time is comparable with the time of annihilation of non-localized Ps (in the recent AMOC experiments for PMMA [10] this trapping time was actually found as $0.54 \pm 0.04 \mathrm{~ns}$ ). This assumption means that some of $o$-Ps atoms annihilate before trapping and was initially based on our published results on Ps annihilation in cross-linked polymers [9] and in porous PPO [6]. In this work, we are trying to find further support of this idea in describing, on the basis of our model, the effect of irradiation on the o-Ps yield in PMMA and PE at low temperatures. However, we did not succeed in this attempt and have to conclude that further investigations are needed to estimate correctly the ratio of the rates of free Ps annihilation and trapping.

\section{Kinetics of Ps trapping}

This problem was considered in previous publications $[6,11]$ for homogeneous distribution of Ps trapping centers (EFV). It was shown also [11] that Ps quenching (without inhibition) by atmospheric oxygen (which is contained not in every trapping center) together with distinct resolution of bimodal distribution of $o$-Ps lifetimes means that Ps atoms moves between the traps, and these EFV are distributed irregularly in polymer matrix. There are microregions with larger and those with smaller EFV. For this case [12]

$$
\begin{array}{ll}
\mathrm{d} P_{\mathrm{f}} / \mathrm{d} t=-\left(\lambda_{\mathrm{f}}^{\mathrm{Ps}}+v_{i}^{\mathrm{t}}\right) P_{\mathrm{f}}, & P_{\mathrm{f}}(0)=3 Q \xi_{i} / 4, \quad i=3,4, \\
\mathrm{~d} P_{\mathrm{loc}} / \mathrm{d} t=v_{i}^{\mathrm{t}} P_{\mathrm{f}}-\gamma_{i} P_{\mathrm{loc}}, & P_{\text {loc }}(0)=0, \\
\xi_{3}=I_{3} /\left(I_{3}+I_{4}\right), & \xi=I_{4} /\left(I_{3}+I_{4}\right) .
\end{array}
$$

Correspondingly

$$
v_{i}^{\mathrm{t}}=\left(4 I_{i} / 3\right)\left(\lambda_{\mathrm{f}}-\gamma_{i}\right) /\left(Q \xi_{i}-4 I_{i} / 3\right), \quad i=3,4 .
$$

$P_{\mathrm{f}}$ and $P_{\text {loc }}$ are the probabilities of finding free or trapped (localized) Ps, $Q$ is the initial fraction of the formed free $\mathrm{Ps}$, and $\lambda_{\mathrm{f}}^{\mathrm{Ps}}$ is corresponding annihilation rate; $\gamma_{i}=1 / \tau_{i}$. We assume that $Q$ and $\lambda_{\mathrm{f}}^{\text {Ps }}$ are the same for the both microphases. On the other hand

$$
v_{i}^{\mathrm{t}}=4 \pi D_{\mathrm{f}}^{\mathrm{Ps}} N_{i} R_{i}
$$

and concentrations of $\mathrm{EFV}, N_{i}$ can be found using previously estimated [6] diffusion coefficient of non-localized Ps $D_{\mathrm{f}}^{\mathrm{Ps}}=0.5 \times 10^{-4} \mathrm{~cm}^{2} / \mathrm{s}$. 


\section{Fractional free volume (FFV)}

Concentration of EFV is very important characteristic of polymers since it determines FFV and permeability of membrane materials. The problem is how can we calculate this characteristic from PAL data. In principle, FFV can be defined as the sum

$$
\mathrm{FFV}=V_{\mathrm{f}}=\sum_{i} v_{\mathrm{f} i} N_{i}=v_{\mathrm{f} 3} N_{3}+v_{\mathrm{f} 4} N_{4}
$$

On the other hand, some authors suggested equation

$$
\mathrm{FFV}=V_{\mathrm{f}}=C\left(v_{\mathrm{f} 3} I_{3}+v_{\mathrm{f} 4} I_{4}\right)
$$

with $C=0.0018$ for glassy polymers [13]. Alternative but more complicated approach could be found in comparison of PAL data and bulk volume-temperature data $[8,14]$. Some aspects of this approach were discussed at the recent positron annihilation conference ICPA12 [15, 16]. The FFV values found by us from PAL measurements are given in Table. For many polymers they are in the range $2-15 \%$

TABLE

Fractional free volume estimated from PAL measurements and permeation $P$ (in barrer) of glassy polymers.

\begin{tabular}{l|c|c|c|c|r}
\hline \hline \multicolumn{1}{c|}{ Polymer } & $\begin{array}{c}\text { FFV } \\
\text { (Bondi) }\end{array}$ & $\begin{array}{c}V_{\mathrm{f} 3}+V_{\mathrm{f} 4} \\
\text { homogeneous }\end{array}$ & $\begin{array}{c}V_{\mathrm{f} 3}+V_{\mathrm{f} 4} \\
\text { heterogeneous }\end{array}$ & $C\left(I_{3} v_{\mathrm{f} 3}+I_{4} v_{\mathrm{f} 4}\right)$ & $\begin{array}{c}P\left(\mathrm{O}_{2}\right) \\
{[\text { barrer }]}\end{array}$ \\
\hline PTMSP & 0.34 & 0.080 & 0.110 & 0.80 & 7700 \\
AF2400 & 0.32 & 0.040 & 0.051 & 0.22 & 1140 \\
AF1600 & 0.28 & 0.020 & 0.025 & 0.13 & 170 \\
PPrSiDPA & 0.12 & 0.065 & 0.120 & 0.62 & 227 \\
PPhSiDPA & 0.15 & 0.016 & 0.034 & 0.11 & 12 \\
PTMSS & & 0.029 & 0.047 & 0.14 & 56 \\
PFPDMSS & & 0.021 & 0.038 & 0.11 & 38 \\
PVTMS & 0.195 & 0.034 & 0.071 & 0.21 & 44 \\
PVPDMS & 0.134 & 0.017 & 0.033 & 0.085 & 2.5 \\
Nafion-117 & & 0.002 & & 0.028 & 1.1
\end{tabular}

and smaller than those calculated in [17] by the Bondi method [18]. This result does not seem to be unexpected: the Bondi method takes into account the whole existing free volume because it is defined as a difference between specific volume and corresponding van der Waals (or occupied) volume, whereas the PAL method should be sensitive to only a part of the size distribution of free volume. However, for high free volume polymers (PTMSP, PPrSiDPA), Eq. (4) gives unrealistic values, as high as $80 \%$, for example in the case of PTMSP. It means that an assumption of a constant and universal value of $C$ for all the glassy polymers may not be correct, and we prefer Eqs. (3), (4) derived from more physical kinetic description (1). They do not give such overestimation. 


\section{Free volume and perm-selectivity}

It is interesting to check an ability of free volume found via PAL data to determine transport properties of polymers. Table permits to compare the permeability coefficient $P$, barrer $\left(1\right.$ barrer $\left.=10^{-10} \mathrm{~cm}^{3}(\mathrm{STP}) \cdot \mathrm{cm} /\left(\mathrm{cm}^{2} \cdot \mathrm{s} \cdot \mathrm{cm} \mathrm{Hg}\right)\right)$ for oxygen with the FFV calculated as $v_{\mathrm{f} 3}+v_{\mathrm{f} 4}$ for a number of polymer glasses. The results show that there exists a strong correlation between PAL distribution, free volume, and permeability. However, another property is of even more importance for membrane materials, their perm-selectivity (permeation selectivity) or ideal separation factor

$$
\alpha_{12}=P_{1} / P_{2}
$$

where $P_{1}$ and $P_{2}$ are low pressure limits of permeability coefficients of gases $M_{1}$ and $M_{2}$. As the permeability coefficient is the product of diffusion and solubility coefficients, solubility and diffusion contributions can be distinguished in the separation factor $\alpha_{12}$, that is

$$
\alpha_{12}=\alpha_{12}^{\mathrm{S}} \alpha_{12}^{\mathrm{D}} \text {. }
$$

It is known that perm-selectivity for light gases in conventional glassy polymers is determined mainly by their diffusion selectivity. In our previous study [17] we had found a dependence of diffusion coefficients for different gases [19] in polymers, included in Table, versus square of kinetic diameter $d^{2}$ of diffusing molecules.

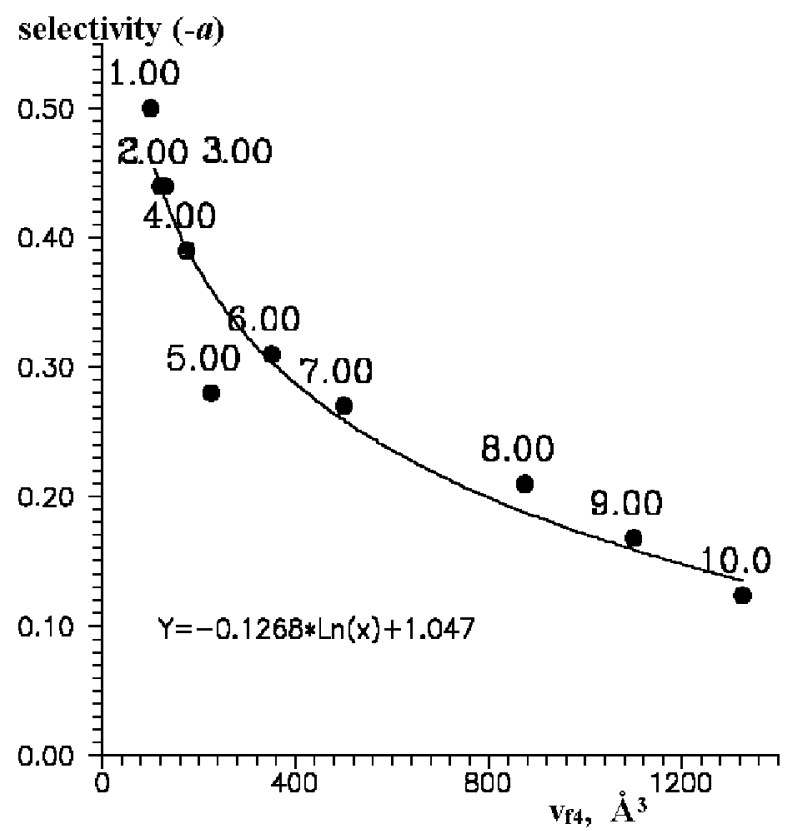

Fig. 1. Diffusion selectivity $(-a)$ as a function of the volume microcavity $v_{\mathrm{f}}=v_{\mathrm{f} 4}\left(\AA^{3}\right)$ : 1 - PSf, 2 - PC, 3 - PS, 4 - PVPDMS, $5-$ PPhSiDPA, 6 - PVTMS, 7 - AF 1600, 8 - AF2400, 9 - PPrSiDPA, 10 - PTMSP. 
It could be seen [17] that the larger diffusion coefficient $D$ in a polymer for a certain gas, the smaller is the slope $a$ of the dependencies of $D$ versus $d^{2}$

$$
\lg D=a d^{2}+b \text {. }
$$

The slope $a$ of these lines determines diffusion selectivity of a polymer. Thus, diffusion selectivity of large free volume polymers like PTMSP and AF2400, is markedly lower than those of conventional glassy polymers such as poly(carbonate) PC. Figure 1 illustrates a dependence of $a$ on $v_{\mathrm{f}}=v_{\mathrm{f} 4}$ determined from the PAL experiments for the polymers of the Table as well as for conventional glassy polymers (poly(sterene) PS, poly(carbonate) PC, poly(sulfone) PSf) [4]. In the last cases, in absence of $v_{\mathrm{f} 4}$, we used $v_{\mathrm{f} 3}$. It is seen that when the size (in $\AA^{3}$ ) of the free volume, determined from the PAL experiments, increases, diffusion selectivity monotonously decreases. This dependence is characteristic only of glassy polymers since rubbers have different mechanism of gas permeation.

\section{Free volume elements or fractional free volume?}

We succeeded in obtaining the dependencies similar to that in Fig. 1 also for $v_{\mathrm{f}}=v_{\mathrm{f} 4}+v_{\mathrm{f} 3}$, but not for $v_{\mathrm{f}}=v_{\mathrm{f} 3}$. It means that namely free volumes of larger radius $R_{4}$ are responsible for gas permeation and selectivity of glassy polymers relative to light gases. Thus, $o$-Ps is sensitive to the free volumes, which are responsible for the membrane properties of glassy polymers. However, what is the character of this dependence? Is it sensitive to the number of EFV or only to their size? In other words, can we actually say that $I_{i}$ is dependent on EFV concentration $N_{i}$ and this concentration can be found from annihilation characteristics $[1,6,13]$, or $I_{i}$ reflects only the ratio between the numbers of EFV of different size and is dependent mostly on the fraction $Q$ of positrons which form Ps $[20,21]$ ? In the first case, o-Ps trapping rate $x_{i}^{\mathrm{t}}(3)$ is comparable with annihilation rate $\lambda_{\mathrm{f}}$ of free $o-\mathrm{Ps}$, while in the second case $x_{i}^{\mathrm{t}} \gg \lambda_{\mathrm{f}}$. Unfortunately, the described experiments still cannot answer this question. Actually, scattering of the data did not permit to make choice between the two cases: $v_{\mathrm{f}}=v_{\mathrm{f} 4}$ and $V_{\mathrm{f}}=v_{\mathrm{f} 4} N_{4}$. This is seen in Fig. 2 and in Fig. 3, where correlations between $v_{\mathrm{f}}, V_{\mathrm{f}}$ and permeability coefficient $P$ (Table) are shown. The reason is in the character of the dependence of $v_{\mathrm{f}}$ and $V_{\mathrm{f}}$ on $R: v_{\mathrm{f} 4} \sim R_{4}^{3}$ while $N_{4} \sim v_{4} / R_{4}$ (see Eq. (3)), and permeation is determined mostly, at least in the studied samples, by the free volume size, but not by the number of these volumes. On the other hand, there are some previously published experiments in the cross-linked polygoether acrylates [9], and poly(diethylene glycol bis(allyl carbonate)) [22], where, for molecules of similar chemical composition (this composition excluded chemical inhibition of Ps formation), intensity of the long-lived component $I_{3}$ was strongly dependent on density of links $c$ : $I_{3}$ went down at higher $c$, so that $I_{3}=0$ was detected for some of compositions [9], and $I_{3}$ went up ( $\tau_{3}$ decreased) for higher $c$ in [22]. Both types of variations of $I_{3}$ can be explained, in principle, by the influence of cross-linking on the number and size of EFV: smaller number of EFV $\left(N_{3}\right)$ for the higher $c$ (for the first type of the dependence) and larger number $N_{3}$ of the holes of smaller size in the second case. Obviously, such cases may be typical of the comparable 


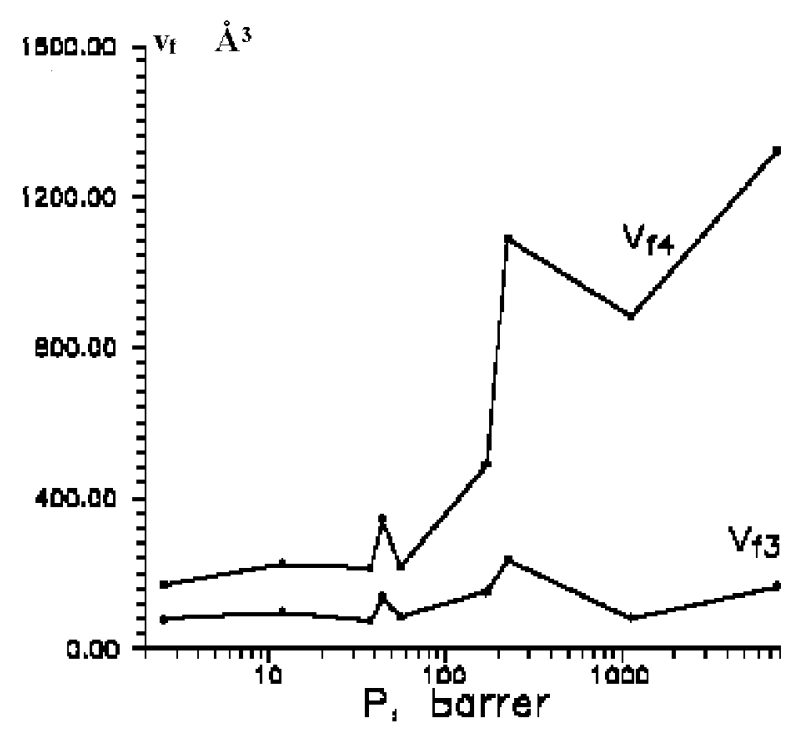

Fig. 2. Correlations between the elementary free volumes $v_{\mathrm{f}}=v_{\mathrm{f} 3}, v_{\mathrm{f} 4}$ and permeability coefficient $P$ for oxygen for the substances shown in Table (excluding nafion).

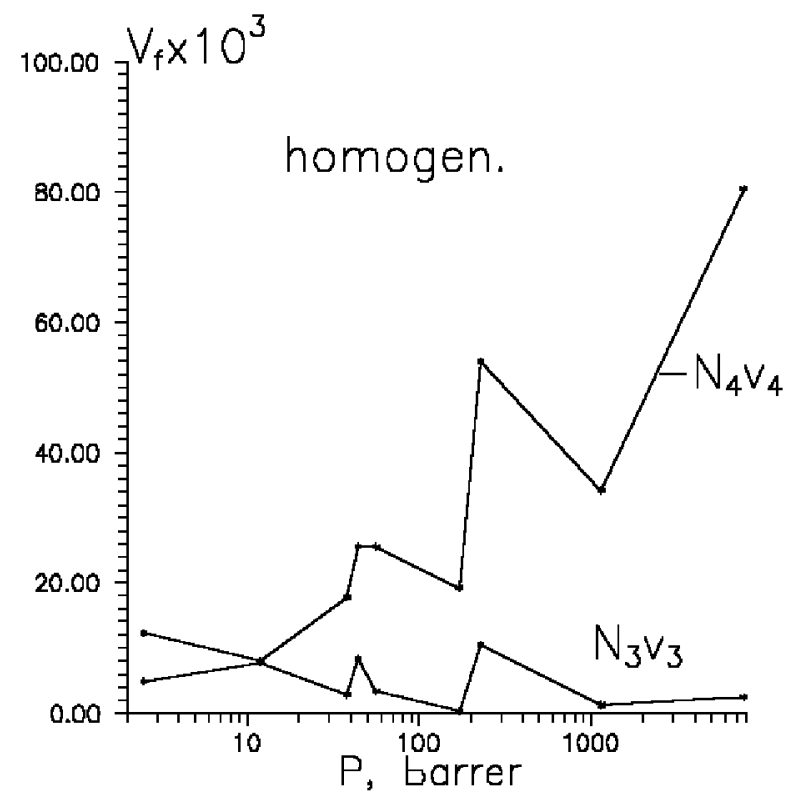

Fig. 3. Correlations between the fractional free volume $V_{\mathrm{f}}$, taken as $v_{\mathrm{f} 3} N_{3}$ or $v_{\mathrm{f} 4} N_{4}$, and permeability coefficient $P$ for oxygen for the substances shown in Table (excluding nafion).

rates $x_{3}$ and $\lambda_{\mathrm{f}}$ and may support the idea that $I_{3}$ is dependent not only on $Q$ but also on EFV concentration $N_{3}$. However, the authors [22] got some arguments showing that $N_{3}$ was not changing in their case. In this uncertain situation we 
were trying [23] to use kinetic consideration of the effect of $\gamma$-irradiation on $o$-Ps formation $\Delta I_{3}$ in PMMA and $\mathrm{PE}$ at low temperatures to put some light on the mechanism of $o$-Ps trapping. The value of $\Delta I_{3}$ was derived as a function of Ps trapping rate $x^{\mathrm{t}}$ and $\mathrm{Ps}$ formation rate $x_{\text {form }}$, the last being dependent on density of the trapped electrons $N_{\mathrm{e}}$

$$
\Delta I_{3}=(3 / 4)(1-Q) A B
$$

$A=v_{\text {form }} /\left(\lambda_{\mathrm{f}}+v_{\text {form }}-\gamma_{3}\right)$ and gives the probability of Ps formation, $B=$ $v^{\mathrm{t}} /\left(\lambda_{\mathrm{f}}+v^{\mathrm{t}}-\gamma_{3}\right)$ is the probability of Ps localization in the Ps trapping sites (EFV). We suppose that $\lambda_{\mathrm{f}}^{+}=\lambda_{\mathrm{f}}^{\text {Ps }} \equiv \lambda_{\mathrm{f}}$ and remember that $\gamma_{3}=1 / \tau_{3}$ and $v_{\text {form }}=k_{\text {form }} N_{\mathrm{e}}=4 \pi D^{+} R N_{\mathrm{e}}$. Fitting of Eq. (9) to experimental points for PE gave $k_{\text {form }}(\mathrm{PE})=10^{-7} \mathrm{~cm}^{3} / \mathrm{s}$, and $B=1$, which actually means that $x^{\mathrm{t}} \gg \lambda_{\mathrm{f}}$ and the probability of Ps trapping in PE is equal to 1. Description of the PMMA results (experimental points in Fig. 4), using Eq. (9), is possible by suggesting the two alternatives:

1. $k_{\text {form }}(\mathrm{PMMA})=(1 / 50) k_{\text {form }}(\mathrm{PE})=0.2 \times 10^{-8} \mathrm{~cm}^{3} / \mathrm{s}, B=1$. This probably means that $D^{+}(\mathrm{PMMA})=(1 / 50) D^{+}(\mathrm{PE})$, where $D^{+}(\mathrm{PE})$ is known to be $0.1 \mathrm{~cm}^{2} / \mathrm{s}$. However, $D^{+}$(PMMA) is unknown.

2. $k_{\text {form }}(\mathrm{PMMA})=k_{\text {form }}(\mathrm{PE})=10^{-7} \mathrm{~cm}^{3} / \mathrm{s}$ and $B=0.2$. Probability of Ps trapping is smaller than 1.

Looking now on Fig. 4, we have to recognize that alternative (1) gives much better description of experimental points for PMMA than the alternative (2). This

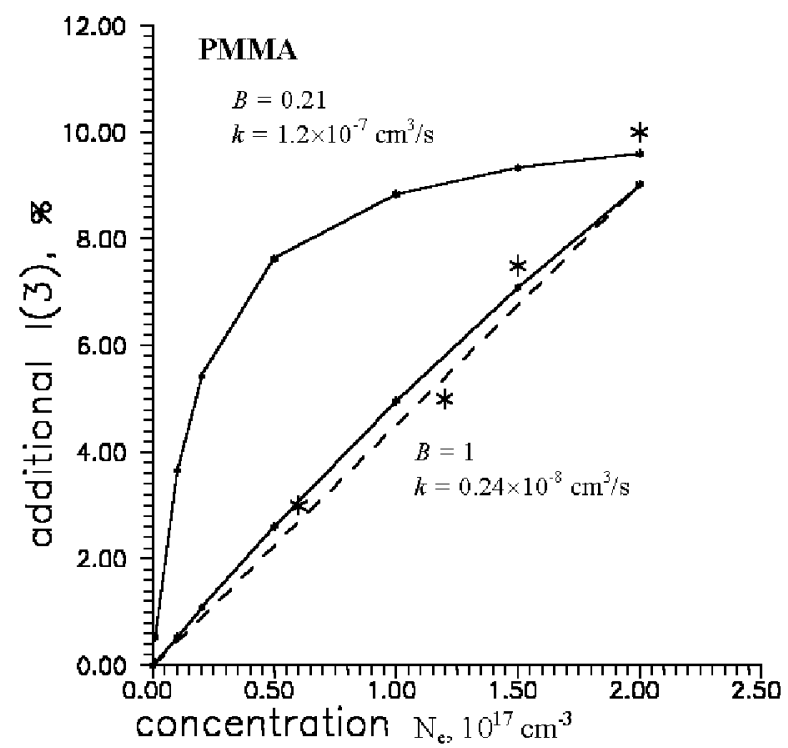

Fig. 4. Additional long-lived component intensity $\Delta I_{3}$ in PMMA as a function of the trapped electron density $N_{\mathrm{e}}$. Solid curves correspond to different sets of parameters in Eq. (9): $k_{\text {form }}(\mathrm{PMMA})=k_{\text {form }}(\mathrm{PE})=1.2 \times 10^{-7} \mathrm{~cm}^{3} / \mathrm{s}, B=0.21$ or $k_{\text {form }}(\mathrm{PMMA})=$ $(1 / 50) k_{\text {form }}(\mathrm{PE})=0.24 \times 10^{-8} \mathrm{~cm}^{3} / \mathrm{s}, B=1$. 
is the first clear argument in favor of the point of view that in such polymers as PE and PMMA $o_{-}$Ps always comes to trapping before annihilation $\left(B=1, x^{\mathrm{t}} \gg \lambda_{\mathrm{f}}\right)$. Further experiments of this type and, may be, also AMOC measurements for other substances could be useful.

\section{Conclusion}

Thus, in spite of the great number of PAL experiments in polymers, there are no final conclusions on the character of the dependence of $o$-Ps long-lived component intensity on the number of elementary free volumes, localizing o-Ps, and on the fraction of positrons, forming Ps. In fact, the problem is reduced to estimation of the ratio of the rates of non-localized $o$-Ps annihilation and trapping. This is directly related to the question on the possibility to estimate fractional free volume, responsible for permeability and selectivity of polymers, directly from PAL experiments. We expect to continue our work in this field.

\section{Acknowledgments}

This work was supported by the Russian Foundation for Basic Research, project no. 98-03-32859, IR 97-1525, and by the INTAS grant No. 97-1936.

\section{References}

[1] W. Brandt, S. Berko, W.W. Walker, Phys. Rev. 120, 1280 (1960).

[2] H.A. Hristov, B. Bolan, A.F. Yee, L. Xie, D.W. Gidley, Macromolecules 29, 8507 (1996).

[3] T. Goworek, Phys. Status Solidi A 102, 511 (1987).

[4] A.V. Goldanskii, V.A. Onischuk, V.P. Shantarovich, V.V. Volkov, Yu.P. Yampolskii, Khim. Fiz. 7, 616 (1988).

[5] O.E. Mogensen, in: Positron Annihilation in Chemistry, Eds. V.I. Goldanskii, F.P. Schaffer, J.P. Toennies, Springer-Verlag, Berlin 1995, p. 155.

[6] V.P. Shantarovich, V.I. Goldanskii, Hyperfine Interact. 116, 67 (1998).

[7] G. Consolati, I. Genco, M. Pegoraro, L. Zanderighi, J. Polym. Sci., Part B: Polym. Phys. 34, 357 (1996).

[8] G. Dlubek, Ch. Hubner, S. Eichler, Phys. Status Solidi A 168, 333 (1998).

[9] V.P. Shantarovich, J. Radioanal. Nucl. Chem., Articles 210, 357 (1996). See also: I.B. Kevdina, Yu.M. Sivergin, V.P. Shantarovich, Khim. Vys. Energ. 30, 145 (1996).

[10] C. Dauwe, N. Balcaen, B. Van Waenberge, S. Van Petegem, H. Stoll, in: Book of Abstracts of the 12th Int. Conf. on Positron Annihilation (ICPA12), München 2000, Rep. O1.

[11] V.P. Shantarovich, Yu.A. Novikov, Z.K. Suptel, I.B. Kevdina, T. Masuda, V.S. Khotimskii, Yu.P. Yampolskii, Radiat. Phys. Chem. 58, 513 (2000).

[12] V.P. Shantarovich, I.B. Kevdina, Yu.P. Yampolskii, Khim. Vys. Energ. 34, 325 (2000) 
[13] X. Hong, Y.C. Jean, H. Yang, S.S. Jordan, W.J. Koros, Macromolecules 29, 7859 (1996).

[14] J.E. Kluin, Z. Yu, S. Vleeshouwers, J.D. McGervey, A.M. Jamieson, R. Simha, Macromolecules 25, 5089 (1992).

[15] J. Kristiak, P. Bandzuch, O. Sausa, J. Zrubcova, J. Bartos, in Ref. [10], Rep. O6.

[16] G. Consolati, in Ref. [10], Rep. IL2.

[17] V.P. Shantarovich, I.B. Kevdina, Yu.P. Yampolskii, A.Yu. Alentiev, Macromolecules, in press.

[18] A. Bondi, J. Chem. Phys. 58, 929 (1954).

[19] The Data Base Gas Separation Parameters of Glassy Polymers, Informregister RF, No. 3585, 1998.

[20] C. Dauve, G. Consolati, J. Kansy, E. Van Waeyenberge, Phys. Lett. A 238, 379 (1998).

[21] T. Hirade, F.H.J. Maurer, M. Eldrup, Radiat. Phys. Chem. 58, 465 (2000).

[22] G. Dlubek, J. Stejny, M.A. Alam, Macromolecules 31, 4574 (1998).

[23] V.P. Shantarovich, T. Hirade, I.B. Kevdina, V.W. Gustov, E.F. Oleinik, Proc. of this Conference, Acta Phys. Pol. A 99, (2001). 